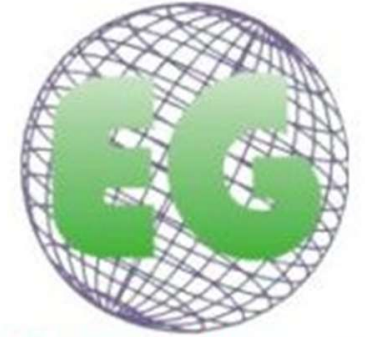

ISSN 1695-6141 $N^{\circ} 59$

\title{
Burnout, habilidades de comunicación y autoeficacia en los profesionales de urgencias y cuidados críticos
}

Burnout, communication skills and self-efficacy in emergency and critical care health professionals

\section{Jesús Llor Lozano ${ }^{1}$}

Ana Myriam Seva Llor ${ }^{1,2}$

José Luis Díaz $\mathrm{Agea}^{3}$

Luis Llor Gutiérrez $z^{1,4}$

César Leal Costa ${ }^{2}$

\author{
${ }^{1}$ Hospital Vega Baja de Orihuela. Alicante, España. \\ ${ }^{2}$ Facultad de Enfermería. Universidad de Murcia.España. \\ ${ }^{3}$ Facultad de Enfermería. Universidad Católica de Murcia. España. \\ 4Departamento de Enfermería. Universidad de Alicante. España. cleal@ucam.edu
}

\section{https://doi.org/10.6018/eglobal.381641}

Recibido: $30 / 05 / 2019$

Aceptado: 18/09/2019

\section{RESUMEN:}

Objetivo: Analizar el nivel y la relación entre habilidades de comunicación, autoeficacia percibida y síndrome de Burnout en profesionales de la salud del servicio de urgencias hospitalario y unidad de cuidados críticos de un hospital comarcal.

Método: Se plantea un estudio observacional y transversal en una muestra de 90 profesionales sanitarios de un Hospital Comarcal, 19 médicos (21,1\%), 47 enfermeros (52,2\%), 24 auxiliares de enfermería $(26,7 \%)$. Se han usado la Escala sobre Habilidades de Comunicación en Profesionales de la Salud, el Maslach Burnout Inventory Human Services Survey y la escala de la Autoeficacia percibida. Resultados: Se obtiene una correlación negativa entre las diferentes dimensiones de la Escala sobre Habilidades de Comunicación en Profesionales de la Salud, la realización personal en el trabajo del Maslach Burnout Inventory y la autoeficacia percibida con las dimensiones agotamiento emocional y despersonalización del Maslach Burnout Inventory. Por otro lado, se observa una correlación positiva y estadísticamente significativa entre las dimensiones de las habilidades de comunicación con la dimensión realización personal en el trabajo del Maslach Burnout Inventory y la autoeficacia percibida.

Conclusiones: Las habilidades de comunicación y la autoeficacia percibida de los profesionales sanitarios en los servicios de urgencias hospitalarios y las unidades de cuidados críticos están relacionadas con un menor agotamiento emocional y despersonalización, y una mayor realización personal en el trabajo.

Palabras clave: Burnout; Comunicación; Autoeficacia; Profesionales de la salud; Urgencias; Cuidados Críticos. 


\begin{abstract}
:
Aim: To analyze the level and relationship between communication skills, perceived self-efficacy and Burnout syndrome in health professionals from the hospital emergency department and the critical care unit of a regional hospital.

Method: An observational and cross-sectional study was conducted with a sample of 90 health professionals from a Regional Hospital, 19 physicians (21.1\%), 47 nurses (52.2\%), 24 nursing aides (26.7\%). The Communication Skills Scale for Health Professionals, the Maslach Burnout Inventory Human Services Survey and the Scale of Perceived Self-Efficacy were utilized.

Results: A negative correlation was obtained between the different dimensions of the Communication Skills Scale for Health Professionals, the personal accomplishment at work of the Maslach Burnout Inventory and the perceived self-efficacy with the emotional exhaustion and depersonalization dimensions of the Maslach Burnout Inventory. On the other hand, a positive and statistically significant correlation was observed between the dimensions of communication skills with the personal accomplishment dimension in the Maslach Burnout Inventory work and the perceived self-efficacy.

Conclusions: The communication skills and the perceived self-efficacy of the health professionals in the emergency department and critical care unit are related to less emotional exhaustion and depersonalization, and greater personal accomplishment at work.
\end{abstract}

Keywords: Burnout; Communication; Self-efficacy; Healthcare professionals; emergency department; Critical Care.

\title{
INTRODUCCIÓN
}

En general, las profesiones sanitarias están íntimamente relacionadas con el estrés laboral; esto genera un problema no solo a nivel individual, sino también a nivel interpersonal o social( ${ }^{(1)}$.

El Síndrome de Burnout se conoce como la respuesta sintomática a situaciones de estrés laboral de tipo crónico que se presenta habitualmente en profesiones asistenciales, además de otras, en las que se requiere un contacto directo constante con las personas. Su manifestación supone un deterioro y un desgaste que afecta a la satisfacción laboral, a la implicación y rendimiento, así como al ámbito personal del individuo. Además de los profesionales de la salud, otro colectivo que sufre este síndrome son los maestros y los deportistas de alto rendimiento ${ }^{(2,3)}$.

Se han detectado tres grandes grupos de factores de riesgo (personales, grupales y organizacionales), relacionados con la aparición de este síndrome. Los factores relacionados con aspectos del ambiente laboral y los factores personales han tenido más relevancia en la mayoría de las investigaciones, siendo los factores de relación con el paciente menos considerados hasta el momento ${ }^{(4)}$.

Este síndrome, fue descrito por Herbert Freudenberger en el año 1974 mientras trabajaba en una clínica para toxicómanos en Nueva York. Allí, observó que en la mayoría de los voluntarios de la clínica había una pérdida de energía progresiva hasta llegar al agotamiento, síntomas de ansiedad y depresión, así como desmotivación en el trabajo y agresividad con los pacientes al cabo de un año de estar trabajando ${ }^{(5)}$.

Otros aspectos que pueden repercutir en el ámbito laboral y en los niveles de burnout en los profesionales sanitarios son las habilidades de comunicación que posean, ya que fomentan el entendimiento mutuo, centrarse en las necesidades del paciente, compartir la responsabilidad y establecer una relación clínica caracterizada por la confianza, el respeto y la empatía(6). Por otro lado, también repercute su estado emocional, su nivel de estrés y su autoeficacia percibida, entendiéndose esta última, como las creencias que las personas poseen sobre sus capacidades, haciendo 
referencia no solo a los recursos de los que realmente se disponga, sino a la opinión profesional que uno tenga sobre lo que se puede hacer con ellos ${ }^{(7)}$.

El personal sanitario se enfrenta a diario a tareas complejas en las que influyen diversos factores estresantes específicos de la profesión que suponen una alta implicación emocional, y los relacionados con la organización del trabajo como, por ejemplo, exceso de estimulación aversiva (enfrentarse frecuentemente a situaciones de sufrimiento, muerte, dolor o pérdida), contacto continuo con enfermos (que exige un cierto grado de implicación para establecer una relación), la frustración de no poder curar, objetivo para el que han sido entrenados, entre otros ${ }^{(1,6)}$.

Por ello, para evitar situaciones de Burnout en el ámbito profesional, los profesionales deberían desarrollar estrategias personales de índole cognitivo-conductual, y con carácter autoevaluativo, para eliminar o reducir fuentes de estrés, tanto a nivel individual como en el grupo de trabajo. Desarrollar unas adecuadas habilidades de comunicación y una percepción de autoeficacia, puede favorecer que el profesional de la salud se sienta más seguro, más competente, fomentando las relaciones interpersonales tanto con sus pacientes como con sus compañeros de trabajo ${ }^{(3,8)}$.

Los servicios de urgencias hospitalarias (SUH) y las unidades de cuidados críticos (UCC), requieren de multitud de tecnologías, cuidados y tratamientos complejos y que hacen que la actividad asistencial se centre en mantener con vida al paciente quedando a un lado la calidad humana. El paciente, la mayoría de veces, se encuentra aislado de sus familiares y amigos, desnudo, lleno de cables y aparatos desconocidos con multitud de alarmas y ruidos, por lo que los profesionales sanitarios, se enfrentan a una enfermedad complicada en situaciones adversas ${ }^{(9,10)}$, desarrollando su labor asistencial en unas particularidades estructurales, emocionales, y de presión asistencial, que hacen que estén expuestos a una mayor cantidad de riesgos y estrés laboral, que pueden favorecer la aparición del síndrome de Burnout ${ }^{(11-17)}$.

Así, como objetivo general del presente estudio nos planteamos analizar la relación de las habilidades de comunicación, autoeficacia percibida y síndrome de Burnout, en profesionales de la salud del servicio de urgencias hospitalario y unidad de cuidados críticos de un hospital comarcal. El objetivo secundario fue analizar las diferencias de puntuaciones de las variables anteriores según las variables sociodemográficas y profesionales.

\section{MÉTODO}

\section{Diseño}

Estudio observacional y transversal llevado a cabo en el Hospital Vega Baja en Alicante, España.

\section{Población y ámbito de estudio}

La muestra se seleccionó mediante muestreo no probabilístico, en el servicio de urgencias hospitalarias y unidad de cuidados críticos. 
Como criterios de inclusión, todos los participantes debían 1) ser mayores de edad, 2) actualmente desempeñar una labor asistencial en el SUH o UCC, 3) ser médico, enfermero o auxiliar de enfermería, y 4) firmar el consentimiento informado.

Para asegurar que la muestra fuese representativa en una población diana de 130 profesionales sanitarios, fue aplicada la siguiente fórmula: $n=\frac{N \cdot Z^{2} \cdot p(1-p)}{(N-1) e^{2}+Z^{2} \cdot p(1-p)}$, con un nivel de confianza $I C=0,95(95 \%)$ una desviación de $Z=1,96$, con un margen de error $e=0,05(5 \%)$, y una proporción de $p=0,05(5 \%)$ como se ha obtenido en estudios previos ${ }^{(18)}$, se obtuvo un tamaño muestral necesario de 47 profesionales, aunque finalmente se consiguieron 90 .

\section{Recogida de datos}

En primer lugar, se solicitó la autorización a la dirección del centro y se contactó con los supervisores de enfermería del SUH y de la UCC. Una vez aceptado por el centro la realización del estudio, se procedió a realizar una entrevista con los supervisores para presentar el proyecto y entregarles el material necesario (cuestionarios on-line y en papel) de los cuestionarios, así como su periodo de cumplimentación.

Se presentaron dos formatos de cuestionario para facilitar la adhesión del personal al estudio, uno en formato digital mediante el uso de Google Formularios, y otro en formato papel.

Al concluir el periodo de recogida de datos, que duró 1 mes y medio, se obtuvo un $90 \%$ de participación (90 cuestionarios cumplimentados), un total de 63 en formato físico y 27 en formato digital. Los cuestionarios en papel fueron recogidos por los supervisores de enfermería de ambas unidades.

\section{Instrumentos}

Para la evaluación los participantes cumplimentaran diversos instrumentos autoadministrados que incluían:

Características sociodemográficas (edad, sexo y estado civil) y profesionales (categoría profesional, tipo de contrato, servicio y tiempo de vida laboral) diseñado ad hoc para el desarrollo del estudio.

Escala sobre Habilidades de Comunicación en Profesionales de la Salud (EHCPS)(19). Escala autoadministrada compuesta por 18 ítems, con una escala de respuesta tipo Likert con 6 grados de respuesta, que está compuesta por cuatro dimensiones: Comunicación informativa: compuesta por 6 ítems $(5,8,9,14,17$ y 18), refleja la manera que los profesionales de la salud tienen de obtener y proporcionar información en la relación clínica que establecen con los pacientes. En esta dimensión la puntuación mínima es de 6 y la máxima de 36. Empatía: compuesta por 5 ítems (2, $4,6,11$ y 12), refleja la capacidad de los profesionales de la salud de comprender los sentimientos de los pacientes y hacerlo evidente en la relación, así como la dimensión conductual de ésta, la actitud empática, compuesta por la escucha activa y la respuesta empática. En esta dimensión la puntuación mínima es de 5 y la máxima de 30. Respeto: compuesta por 3 ítems (1, 3 y 15), evalúa el respeto que muestran los profesionales de la salud en la relación clínica que establecen con los pacientes. En esta dimensión la puntuación mínima es de 3 y la máxima de 18. Habilidad social: los 4 ítems de esta dimensión $(7,10,13$ y 16) reflejan la habilidad que los profesionales 
de la salud tienen de ser asertivos o tener conductas socialmente habilidosas en la relación clínica que establecen con los pacientes. En esta dimensión la puntuación mínima es de 4 y la máxima de 24. Esta herramienta presentó unas adecuadas propiedades psicométricas en cuanto a análisis de ítems, estructura interna, fiabilidad (Alpha de Cronbach fue de 0,65 a 0,78 en todas las dimensiones) y evidencias externas de validez, relacionando las habilidades de comunicación con el nivel de burnout percibido por los profesionales de la salud ${ }^{(20)}$.

Maslach Burnout Inventory Human Services Survey (MBI-HSS), en su versión adaptada a la población española es el más utilizado para evaluar la frecuencia e intensidad del síndrome de Burnout dentro del contexto laboral sanitario. Consta de 22 ítems, y en él se pueden diferenciar tres dimensiones que conforman el síndrome: agotamiento emocional (AE), despersonalización (DP) y realización personal en el trabajo (RP). Estos 22 ítems se valoran con una escala tipo Likert, en la que el sujeto puntúa la frecuencia con la que experimenta los sentimientos que configuran el síndrome. El rango de frecuencia está formado por 7 categorías que van de "nunca" (0) a "todos los días" (6). La puntuación mínima y máxima es de 0-54 en la dimensión $\mathrm{AE}$, de 0-30 en la dimensión DP, y de 0-48 en la dimensión RP. La consistencia interna ( $\alpha$ de Cronbach) fue satisfactoria para las dimensiones $A E(\alpha=0,85)$ y $\operatorname{RP}$ $(\alpha=0,71)$, y moderada para la dimensión DP $(\alpha=0,58)$. La estructura de tres factores oblicuos presentó un buen ajuste(21).

Cuestionario de Autoeficacia, que consta de 10 ítems que engloban en sí misma la dimensión de la autoeficacia laboral y evalúa la creencia estable de la gente sobre su capacidad para manejar adecuadamente una amplia gama de estresores de la vida cotidiana. La puntuación mínima y máxima la escala es de 10 y 40 . La escala mostró una consistencia interna $(\alpha)$ de 0,87 y una correlación dos mitades de $0,88^{(22)}$.

\section{Análisis de datos}

A la hora del procesamiento de datos, en primer lugar se realizó una base de datos en formato Microsoft Excel $®$ donde se introdujeron todos los cuestionarios recogidos, se codificaron las variables y posteriormente se analizaron en el programa SPSS@ v23, realizándose las siguientes análisis: estadísticos descriptivos (media, desviación estándar) de las diferentes puntuaciones obtenidas por los profesionales de la salud en las variables cuantitativas (dimensiones de los diferentes instrumentos y edad), así como frecuencias y porcentajes de las variables sociodemográficas y profesionales categóricas. Por otro lado, se realizaron correlaciones bivariadas de Pearson entre las variables estudiadas y diferencias de medias (t-Student y ANOVA de un factor) entre las diferentes dimensiones del MBI, la EHC-PS y la autoeficacia percibida con la variable sociodemográfica sexo y las variables profesionales categoría profesional y ámbito de trabajo. Para finalizar, en los ANOVAS que resultaron significativos se aplicó un análisis post-hoc de Tukey, para identificar las diferencias significativas entre los pares de la variable categoría profesional.

Antes de realizar la prueba t-Student y el ANOVA de un factor se comprobó el supuesto de normalidad de las variables a través del test de Kolmogorov-Smirnov, y la homocedasticidad de las varianzas, utilizando el test de Levene.

\section{Consideraciones éticas}

Para la realización del estudio, se siguieron las normas de Protección de Datos de Carácter Personal que se establecen para los datos de salud(23), y recomendaciones 
de ámbito internacional: garantía de participación voluntaria e informada, confidencialidad de los datos, e información a los participantes de los resultados. Para este fin, se dieron instrucciones para que pusieran un "Alias" anónimo en los cuestionarios escritos para su posterior análisis.

\section{RESULTADOS}

La muestra final estuvo compuesta por 90 profesionales de la salud, de los cuales, 47 eran enfermeros $(52,2 \%), 19$ eran médicos $(21,1 \%)$ y 24 eran auxiliares de Enfermería $(26,7 \%)$.

La media de edad de la muestra fue de 42 (DE=7,40) años, siendo el $27,8 \%$ hombres y el $72,2 \%$ mujeres. En la tabla 1 se muestran los estadísticos descriptivos de las variables sociodemográficas y profesionales de la muestra (Tabla 1).

El 52,2\% ( $n=47)$ de los profesionales de la salud trabajaban en el SUH, y el 47,8\% $(n=43)$ en la UCC, obteniendo una tasa de respuesta adecuada en ambas unidades, siendo del $67,1 \%$ en el SUH y del $71,7 \%$ en la UCC.

Se encontraron unas puntuaciones medias-altas en todas las dimensiones de la EHCPS, y una puntuación alta en la dimensión realización personal en el trabajo del MBIHSS y autoeficacia percibida. Por otro lado, se obtuvieron puntuaciones medias en la dimensión asertividad y la dimensión agotamiento emocional del MBI-HSS, y bajas en la dimensión despersonalización del MBI-HSS (Tabla 1).

Tabla 1. Estadísticos descriptivos de las variables socio-demográficas, profesionales y de las dimensiones de los instrumentos utilizados.

\begin{tabular}{|l|c|}
\hline Variables sociodemográficas & $\mathbf{n ~ ( \% )}$ \\
\hline Sexo & \\
Hombre & $25(27,8)$ \\
Mujer & $65(72,2)$ \\
\hline Estado civil & \\
Soltero/a & $27(30)$ \\
Casado/a & $55(61,1)$ \\
Divorciado/a & $7(7,8)$ \\
Viudo/a & $1(1,1)$ \\
\hline Tipo de contrato & \\
Indefinido & $29(32,2)$ \\
Temporal & $54(60)$ \\
Reducción de jornada & $5(5,6)$ \\
Formación y aprendizaje & $2(2,2)$ \\
\hline Categoría Profesional & \\
Enfermería & $47(52,2)$ \\
Medicina & $19(21,1)$ \\
Auxiliar de Enfermería & $24(26,7)$ \\
\hline Tiempo Trabajado & \\
3 meses a 1 año & $0(0)$ \\
1-5 años & $0(0)$ \\
5-10 años & $4(4,5)$ \\
> de 10 años & $10(11,1)$ \\
\hline
\end{tabular}




\begin{tabular}{|l|c|}
\hline $\begin{array}{c}\text { Servicio } \\
\text { Urgencias }\end{array}$ & $47(52,2)$ \\
UCC & $43(47,8)$ \\
\hline Edad (años) [M (DE)] & $42(7,40)$ \\
\hline Dimensiones & $\mathbf{M}(\mathrm{DE})$ \\
\hline Escala sobre Habilidades de Comunicación & \\
(EHC-PS) & $27,36(4,46)$ \\
Comunicación informativa & $23,18(4,05)$ \\
Empatía & $15,61(2,12)$ \\
Respeto & $15,84(3,73)$ \\
Habilidad Social & \\
\hline Maslach Burnout Inventory (MBI-HSS) & $30,69(11,5)$ \\
Cansancio emocional & $13,48(6,01)$ \\
Despersonalización & $45,98(8,91)$ \\
Realización personal & $32,14(4,15)$ \\
\hline Autoeficacia Percibida & \\
\hline
\end{tabular}

En cuanto al análisis de la comparación de medias, se pude observar que los hombres puntúan más alto que las mujeres en todas las dimensiones de la EHC-PS excepto en la dimensión empatía, en realización personal en el trabajo del MBI-HSS y en autoeficacia general, sin embargo, estas diferencias no son estadísticamente significativas $(p>0,05)$ (Tabla 2).

Tabla 2. Estadísticos descriptivos (media, desviación estándar) de las puntuaciones de la muestra en cada una de las dimensiones de la EHC-PS y del MBI-HSS según la variable sexo.

\begin{tabular}{lccccc}
\hline & \multicolumn{3}{c}{ Hombre } & \multicolumn{3}{c}{ Mujer } \\
& \multicolumn{2}{c}{$\mathrm{n}=25$} & $\mathrm{n}=65$ \\
\hline & $\mathrm{M}$ & $\mathrm{DE}$ & $\mathrm{M}$ & $\mathrm{DE}$ & $\mathrm{p}$ \\
Empatía (EHC-PS) & 22,92 & 4,05 & 23,27 & 4,07 & 0,71 \\
Comunicación Informativa (EHC- & 28,28 & 4,14 & 27 & 4,55 & 0,22 \\
PS) & & & & & \\
Respeto (EHC-PS) & 16,16 & 1,72 & 15,40 & 2,23 & 0,13 \\
Habilidad Social (EHC-PS) & 16,28 & 3,9 & 15,67 & 3,68 & 0,49 \\
Agotamiento Emocional (MBI- & 30,04 & 12,67 & 30,93 & 11,12 & 0,74 \\
HSS) & 13,96 & 6,45 & 13,29 & 5,87 & 0,64 \\
Despersonalización (MBI-HSS) & 47,04 & 7,68 & 45,56 & 9,36 & 0,49 \\
RPT (MBI-HSS) & 33,24 & 3,57 & 31,72 & 4,30 & 0,12 \\
Autoeficacia & \multicolumn{5}{c}{. } \\
\hline M: Media; DE: Desviación Estándar; p: Significación; RPT: Realización personal en el trabajo
\end{tabular}

Si nos fijamos en las variables según la unidad, observamos que los profesionales de la salud de la UCC obtuvieron una mayor puntuación en todas las dimensiones de la EHC-PS con respecto a los del SUH, siendo estas diferencias estadísticamente significativas en las dimensiones empatía (t $88=4,158 ; p=0,000)$, comunicación informativa (t $\left.\mathrm{t}_{8}=2,520 ; p=0,014\right)$ y asertividad $\left(\mathrm{t}_{88}=2,481 ; p=0,015\right)$. En las dimensiones agotamiento emocional y despersonalización, el personal de la UCC obtuvo menores puntuaciones, siendo las puntuaciones similares en las demás dimensiones y la autoeficacia percibida (Tabla 3). 
Tabla 3. Estadísticos descriptivos (media, desviación estándar) de las puntuaciones de la muestra en cada una de las dimensiones de la EHC-PS y del MBI-HSS según la variable servicio.

\begin{tabular}{lccccc}
\hline & \multicolumn{2}{c}{ Urgencias } & \multicolumn{2}{c}{ UCC } \\
& $\mathrm{n}=47$ & $\mathrm{n}=43$ & \\
\hline & $\mathrm{M}$ & $\mathrm{DE}$ & $\mathrm{M}$ & $\mathrm{DE}$ & $\mathrm{p}$ \\
Empatía (EHC-PS) & 21,61 & 4,36 & 24,88 & 2,85 & 0,00 \\
Comunicación Informativa (EHC- & 26,25 & 4,77 & 28,55 & 3,78 & 0,01 \\
PS) & & & & & \\
Respeto (EHC-PS) & 15,34 & 2,35 & 15,90 & 1,82 & 0,21 \\
Habilidad Social (EHC-PS) & 14,93 & 4,09 & 16,83 & 3,03 & 0,01 \\
Agotamiento Emocional (MBI- & 32,04 & 10,95 & 29,20 & 12,03 & 0,24 \\
HSS) & & & & & \\
Despersonalización (MBI-HSS) & 14,17 & 6,55 & 12,72 & 5,32 & 0,25 \\
RPT (MBI-HSS) & 46,12 & 9,74 & 45,81 & 8,00 & 0,87 \\
Autoeficacia & 32,77 & 3,60 & 31,46 & 4,63 & 0,14 \\
\hline M: Media; DE: Desviación Estándar; p: Significación; RPT: Realización personal en el trabajo
\end{tabular}

El análisis de la comparación de medias según la variable categoría profesional, arrojó diferencias estadísticamente significativas en las dimensiones comunicación informativa $\left(F_{2,87}=4,872 ; p=0,010\right)$, respeto $\left(F_{2,87}=3,867 ; p=0,025\right)$, y asertividad $\left(F_{2,87}=3,503 ; p=0,034\right)$. El análisis Post Hoc mostró que estas diferencias eran entre médico y enfermeras con los técnicos en cuidados auxiliares de enfermería en las dimensiones comunicación informativa y respeto, y, por otro lado, entre enfermeras y técnicos en cuidados auxiliares de enfermería en la dimensión asertividad. En cuanto a la autoeficacia general percibida, aunque las puntuaciones son similares, el colectivo que más alto puntuó fue el de las enfermeras, seguidas de técnicos en cuidados auxiliares de enfermería y médicos respectivamente (Tabla 4).

Tabla 4. Estadísticos descriptivos (media, desviación estándar) de las puntuaciones de la muestra en cada una de las dimensiones de la EHC-PS y del MBI-HSS según la variable categoría profesional.

\begin{tabular}{ccc} 
Médicos $^{a}$ & $\begin{array}{c}\text { Enfermeras }^{b} \\
(n=19)\end{array}$ & $\begin{array}{c}\text { T. C Auxiliares de } \\
\text { enfermería } \\
(n=47)\end{array}$ \\
\hline
\end{tabular}

\begin{tabular}{|c|c|c|c|c|c|c|c|}
\hline & $M$ & $\overline{\mathrm{DE}}$ & $\mathrm{M}$ & $\overline{D E}$ & $\mathrm{M}$ & $\mathrm{DE}$ & $p$ \\
\hline Empatía (EHC-PS) & 24,32 & 4,44 & 23,47 & 3,57 & 21,71 & 4,36 & 0,08 \\
\hline $\begin{array}{l}\text { Comunicación } \\
\text { Informativa (EHC-PS) }\end{array}$ & $28,53^{c}$ & 3,73 & $28,06^{c}$ & 3,49 & $25,04^{a, b}$ & 5,83 & 0,01 \\
\hline Respeto (EHC-PS) & $16,16^{c}$ & 1,83 & $15,89^{c}$ & 2,08 & $14,63^{a, b}$ & 2,18 & 0,02 \\
\hline $\begin{array}{l}\text { Habilidad Social } \\
\text { (EHC-PS) }\end{array}$ & 16,58 & 3,81 & $16,40^{\mathrm{c}}$ & 3,13 & $14,17^{b}$ & 4,36 & 0,03 \\
\hline $\begin{array}{l}\text { Agotamiento } \\
\text { Emocional (MBI-HSS) }\end{array}$ & 27,79 & 12,62 & 32,85 & $\begin{array}{c}10,8 \\
1\end{array}$ & 28,75 & 11,55 & 0,17 \\
\hline $\begin{array}{l}\text { Despersonalización } \\
\text { (MBI-HSS) }\end{array}$ & 12,74 & 5,00 & 14,02 & 6,18 & 13,00 & 6,53 & 0,66 \\
\hline RPT (MBI-HSS) & 43,53 & 8,27 & 47,17 & 6,90 & 45,58 & 12,27 & 0,31 \\
\hline Autoeficacia & 31,32 & 3,23 & 32,72 & 3,89 & 31,67 & 5,16 & 0,37 \\
\hline
\end{tabular}

El análisis de diferencias de medias en las demás variables sociodemográficas (estado civil) y profesionales (tipo de contrato y tiempo de vida laboral) no arrojó diferencias estadísticamente significativas $(p>0,05)$. 
En cuanto al análisis de las correlaciones bivariantes, se observó una correlación negativa entre las dimensiones Agotamiento emocional y Despersonalización del MBIHSS y las dimensiones de la EHC-PS, la realización personal en el trabajo del MBIHSS y la autoeficacia percibida. Esta relación fue estadísticamente significativa $(p<0,05)$ entre las dimensiones autoeficacia percibida y agotamiento emocional, $y$, por otro lado, entre las dimensiones empatía, respeto y autoeficacia percibida con despersonalización.

Por otro lado, se observa una correlación positiva y estadísticamente significativa $(p<0,005)$ entre la dimensión realización personal en el trabajo del MBI-HSS y las dimensiones de las habilidades de comunicación en los profesionales de la salud y la autoeficacia percibida (Tabla 5).

Tabla 5. Correlaciones bivariadas de Pearson entre las variables sociodemográficas (edad, sexo, y tiempo trabajado), las dimensiones de las habilidades de comunicación (Comunicación informativa, empatía, respeto y asertividad), las dimensiones del burnout (agotamiento emocional, despersonalización y realización personal) y la Autoeficacia percibida.

\begin{tabular}{lllllllllll}
\hline & Edad & TVL & Cl & E & R & HS & CE & D & RPT & AE \\
\hline Edad & 1 & & & & & & & & & \\
TVL & $0,44^{* *}$ & 1 & & & & & & & & \\
CI & 0,02 & 0,07 & 1 & & & & & & & \\
E & $-0,03$ & $-0,05$ & $0,60^{* *}$ & 1 & & & & & & \\
R & $-0,10$ & $-0,02$ & $0,51^{* *}$ & $0,52^{* *}$ & 1 & & & & & \\
HS & $-0,09$ & 0,01 & $0,60^{* *}$ & $0,63^{* *}$ & $0,23^{*}$ & 1 & & & & \\
CE & $-0,13$ & 0,01 & $-0,13$ & $-0,13$ & $-0,16$ & 0,06 & 1 & & & \\
D & $-0,20$ & $-0,09$ & $-0,16$ & $-0,26^{*}$ & $-0,21^{*}$ & $-0,03$ & $0,62^{* *}$ & 1 & & \\
RPT & $-0,11$ & $-0,01$ & $0,31^{* *}$ & $0,40^{* *}$ & $0,31^{* *}$ & 0,21 & $-0,09$ & $-0,14$ & 1 & \\
AE & $-0,05$ & 0,04 & 0,15 & 0,05 & 0,04 & 0,11 & $-0,34^{* *}$ & $-0,15$ & $0,35^{* *}$ & 1 \\
\hline
\end{tabular}

TVL= Tiempo de vida laboral; Cl= Comunicación Informativa (EHC-PS); E= Empatía (EHC-PS); R= Respeto (EHC-PS); HS= Habilidad Social (EHC-PS); CE = Cansancio emocional (MBI-HSS); D= Despersonalización (MBI-HSS); RPT: Realización personal en el trabajo (MBI-HSS); AE = Autoeficacia.

** La correlación es significativa en el nivel 0,01.

* La correlación es significativa en el nivel 0,05.

\section{DISCUSIÓN}

En este estudio se planteó analizar la relación entre habilidades de comunicación, síndrome de Burnout y autoeficacia en una muestra heterogénea compuesta por tres colectivos profesionales de nuestro sistema sanitario (médicos, enfermeros y auxiliares de enfermería) y de dos de los servicios donde más se aprecia el síndrome de Burnout y la necesidad de entrenar habilidades de comunicación entre los profesionales y los pacientes, como son el SUH y UCC.

La muestra ha obtenido una puntuación media-alta en todas las dimensiones de las habilidades de comunicación, siendo los médicos y los enfermeros los colectivos profesionales con puntuaciones más altas. El colectivo que menos puntuó en habilidades de comunicación fue el de técnicos en cuidados auxiliares de enfermería. Estas diferencias fueron estadísticamente significativas. Estos resultados son coherentes con otros estudios similares ${ }^{(3,24)}$.

Por otro lado, cabe destacar que los profesionales de la salud de la UCC obtuvieron unas mayores habilidades de comunicación con respecto a los del SUH, siendo estas 
diferencias estadísticamente significativas en empatía, comunicación informativa y asertividad. Sin embargo, las puntuaciones en burnout y autoeficacia percibida fueron similares. En cuanto al análisis de las puntuaciones según la variable sexo, generalmente los hombres obtuvieron mayores puntuaciones en habilidades de comunicación, excepto en empatía, realización personal en el trabajo y autoeficacia, y los mismos niveles, en las dimensiones agotamiento emocional y despersonalización.

Podemos considerar que estos resultados son consecuencia directa no solo de la formación en las distintas habilidades profesionales descritas, sino también de la implicación directa de la institución en el fomento de un adecuado contexto laboral.

Una posible solución al problema del estrés entre profesionales sanitarios fue argumentada por en un estudio(4) en el que se afirmaba que la incorporación de manera sistemática de un conjunto de conocimientos, recursos y habilidades comunicativas, podría prevenir el síndrome de burnout. Así, la realización programas de formación donde se aprendan recursos, como por ejemplo el entrenamiento en habilidades de comunicación, manejo de la autoestima, técnicas de relajación, técnicas de afrontamiento, etc., podría incidir de forma positiva en la prevención de este síndrome. Según nuestros resultados, el colectivo que más bajo han puntuado en habilidades de comunicación y más alto en agotamiento emocional y despersonalización, es el de técnicos en cuidados auxiliares de enfermería, quienes podrían beneficiarse ampliamente de este tipo de programas.

Un estudio(24) en el análisis del Burnout en una muestra de enfermeros de emergencias y cuidados críticos de un hospital de la comunidad de Andalucía (España), afirma que la muestra obtiene unas puntuaciones bajas en agotamiento emocional y despersonalización, y elevadas en realización personal en el trabajo, no habiendo niveles altos de burnout, coincidiendo con nuestros resultados.

Asimismo, otro estudio(25) realizado en médicos del SUH, afirma que la autoeficacia, tiene un papel fundamental transformando las emociones en el trabajo. Con lo que se debería de orientar las intervenciones hacia la potenciación de la autoeficacia, a la hora de diseñar programas de promoción de salud laboral.

Por otro lado, los resultados de nuestro trabajo, están en la línea de otro estudio ${ }^{(3)}$ con una muestra de profesionales sanitarios (médicos, enfermeros y auxiliares de enfermería) de varios hospitales, tanto públicos como privados, en las áreas de atención primaria y atención especializada, en el cual, se evidenció de forma empírica que las habilidades de comunicación del personal sanitario son un factor preventivo del síndrome de Burnout. Así, los resultados mostrados en el análisis de correlaciones bivariantes de nuestro estudio son congruentes con otros estudios ${ }^{(3,26)}$ observando una relación negativa entre las habilidades de comunicación y las dimensiones agotamiento emocional y despersonalización, y una relación positiva entre habilidades de comunicación y la realización personal en el área laboral.

Como limitaciones del estudio, podemos argumentar que se podrían de haber introducido otras variables predictoras (como la presión laboral, la autoestima, el estrés, entre otras). Así en próximas investigaciones se podrá dar un paso más e incluir más dimensiones que expliquen las particularidades y especificidades del Burnout en los profesionales de la salud de los SUH y las UCC.

Otras limitaciones importantes fueron que el muestro no fue aleatorio, no se estratificó la muestra ni en función del servicio, ni en función del tipo de profesional, y que la 
muestra fue pequeña, a pesar de contar con una gran participación de los profesionales. Así, en próximos estudios se podría aumentar la muestra a profesionales de otros centros sanitarios, realizando un estudio multicéntrico, estratificando la muestra según el servicio y la categoría profesional, asegurando así, que la muestra fuera representativa en las distintas categorías profesionales.

\section{CONCLUSIONES}

Con los resultados obtenidos surgen varias conclusiones, los profesionales de la salud de la muestra analizada tienen unas adecuadas habilidades de comunicación, una elevada realización personal en el trabajo y autoeficacia percibida. Por otro lado, tienen un bajo nivel de agotamiento emocional y despersonalización. Los profesionales que más habilidades de comunicación tienen son los médicos y las enfermeras, siendo el colectivo de técnicos en cuidados auxiliares de enfermería los que menos. Los hombres tienen mayores niveles de habilidades de comunicación, excepto en empatía, realización personal en el trabajo y autoeficacia que las mujeres, y los mismos niveles de agotamiento emocional y despersonalización. Los profesionales de la salud de la UCC tienen unas mayores habilidades de comunicación, y menor agotamiento emocional y despersonalización, con respecto a los del SUH. Las habilidades de comunicación de los profesionales sanitarios en los SUH y las UCC están relacionadas con un menor agotamiento emocional y despersonalización, y una mayor realización personal en el trabajo, así como el aumento de la percepción de la autoeficacia adquirida.

\section{REFERENCIAS}

1. Gil-Monte PR. El síndrome de quemarse por el trabajo (burnout) Una Enfermedad Laboral en la Sociedad del Bienestar. Madrid: Pirámide; 2005.

2. Moreno-Jiménez B, González JL, Garrosa E. Desgaste profesional (burnout), Personalidad y Salud percibida. En Buendía J, Ramos F. Empleo, estrés y salud. Madrid: Pirámide; 2001: 59-83.

3. Leal-Costa C, Díaz-Agea JL, Tirado-González S, Rodríguez-Marín J, Van-der Hofstadt CJ. Las habilidades de comunicación como factor preventivo del síndrome de Bournout en los profesionales de la salud. An Sist Sanit Navar. 2015;38(2):213-23. Disponible en: https://recyt.fecyt.es//index.php/ASSN/article/view/31787/21785 article/view/31787/21785

4. Cañadas-De la Fuente GA, Vargas C, San Luis C, García I, Cañadas GR, De la Fuente El. Risk factors and prevalence of burnout syndrome in the nursing profession. Int J Nurs Stud. 2015; 52: 240-9

5. Castillo-Ávila IY, Orozco JC, Alvis E. LR. Síndrome de Burnout en el personal médico de una institución prestadora de servicios de salud de Cartagena de Indias. Burn Syndr Med Pers a Heal Serv Provid Cart. 2015;47(2):187-92. Disponible en: http://search.ebscohost.com/login.aspx?direct=true\&db=fua\&AN=103390909\&lang=es \&site=ehost-live

6. Lucas-Milán MG, Moreno-Manso JM y A Suárez-Muñoz. Comunicación interpersonal e incidencia del burnout en la interacción entre sanitarios y pacientes. Apuntes de Psicología. 2015; 33 (1): 23-29.

7. Bandura, A. Pensamiento y acción. Martinez Roca. Barcelona, 1987.

8. Fuertes Goñi C, Aranda Auserón G, Arroyo Anies MP. Comunicación y mindfulness como prevención del burnout. An Sist Sanit Navar. 2016;39(2):331-3. 
9. Pérez-Fuentes MC, Molero-Jurado MM, Gázquez-Linares JJ y MM Simón-Márquez. Análisis de los predictores del burnout en enfermería: factores psicológicos de riesgo y protección. The European Journal of Psychology Applied to Legal Context. 2019; 11 (1) 33-40.

10. Escudero D, Viña L, Calleja C. Por una UCI de puertas abiertas, más confortable y humana. Es tiempo de cambio. Med Intensiva. 1 de agosto de 2014; 38(6):371-5. Disponible en: http://linkinghub.elsevier.com/retrieve/pii/S0210569114000370

11. Carrillo-García C, Ríos-Rísquez MI, Martínez-Hurtado R y P Noguera-Villaescusa. Nivel de estrés del personal de enfermería de la Unidad de Cuidados Intensivos de un hospital clínico universitario. Enfermería Intensiva. 2016; 27 (3): 89-95

12. Fernández Araque A, Cuairán Sola M, Curbelo Rodríguez, R. Calidad de vida profesional de enfermería en urgencias de un hospital de referencia. Enfer Glob 2016; 42: $376-385$.

13. Gómez-Martínez S, Ballester-Arnal R, Gil-Julia B y R Abizanda-Campos. Ansiedad, depresión y malestar emocional en los profesionales sanitarios de las Unidades de Cuidados Intensivos. Anales de Psicología. 2015; 31, (2): 743-750.

14. Julián-Jiménez A, Lizcano-Lizcano A, Carrasco-Vidoz CA y S. Estébanez-Seco. Residentes, guardias en el servicio de Urgencias y síndrome de Burnout. An. Sist. Sanit. Navar. 2015; 38 (3): 453-455.

15. Guerrero-Barona E, García-Baamonde E, Moreno-Manso JM y P González-Rico. Estrés laboral e inteligencia emocional en el servicio de urgencias y emergencias 112. Emergencias 2016; 28:353-355.

16. Sánchez Alonso P y Sierra Ortega, VM. Síndrome de Burnout en el personal de enfermería en UVI. Enfer Glob 2014; 33: 252-266.

17. Nespereira-Campuzano T y Vázquez-Campo M. Inteligencia emocional y manejo del estrés en profesionales de Enfermería del Servicio de Urgencias hospitalarias. Enferm Clin. 2017; 27 (3): 172-178.

18. Ríos Risquez MI, Godoy Fernández C, Peñalver Hernández F, Alonso Tovar AR, López Alcaraz F, López Romera A, et al. Estudio comparativo del burnout en personal de enfermería de Cuidados Intensivos y Urgencias. Enfermería Intensiva. 1 de enero de 2008;19(1):2-13.

19. Leal-Costa C, Tirado-González S, van-der Hofstadt CJ, Rodríguez-Marín J. Creación de la Escala sobre Habilidades de Comunicación en Profesionales de la Salud, Introducción Fase I: Definición del constructo a evaluar y relaciones entre sus componentes y otros constructos externos. Juicio de expertos. An Psicol. 2016;32(1):49-59. Disponible en: http://dx.doi.org/10.6018/analesps.31.3.184701

20. Leal-Costa C, Tirado-González S, Rodríguez-Marín J, vander-Hofstadt-Román CJ. Psychometric properties of the Health Professionals Communication Skills Scale (HPCSS). Int J Clin Heal Psychol. 2016;16(1):76-86. Disponible en: http://dx.doi.org/10.1016/j.ijchp.2015.04.001

21. Gil-Monte PR. Factorial validity of the Maslach Burnout Inventory (MBI-HSS) among Spanish professionals Validação fatorial de Maslach Burnout Inventory (MBIHSS) para profissionais espanhóis. Rev Saúde Pública. 2005;39(1):1-8. Disponible en: www.fsp.usp.br/rsp

22. Sanjuán-Suárez $P$, Pérez-García AM, Bermúdez-Moreno J. Escala de autoeficacia general: Datos psicométricos de la adaptación para población española. Psicothema. 2000;12(SUPPL. 2):509-13.

23. Ley Orgánica $3 / 2018$, de 5 de diciembre, de Protección de Datos Personales y garantía de los derechos digitales. BOE. 2018; 294: 119788 - 119857.

24. Sánchez Romero, S. Prevalencia del Sindrome de Burnout en profesionales de Enfermeria de las Unidades de Cuidados Críticos y Urgencias del Hospital MédicoQuirúrgico de Jáen. Enfermería del Trabajo. 2014; 4: 115-125. 
25. Castaño MB, Gelaber M, Terol-Cantero MC. La autoeficacia y su relación con la salud psicosocial ocupacional en médicos de urgencias hospitalarias. UCMalue, Revista Académica. 2017;75-91.

26. Yuguero O, Forné $\mathrm{C}$, Esquerda M, Pifarré J, Abadías MJ, Viñas J. Empathy and burnout of emergency professionals of a health region: A cross-sectional study. Medicine (Baltimore). 2017;96(37): e8030. 